\title{
Novel Immunotherapeutics for the Treatment of Glioblastoma: The Last Decade of Research
}

\author{
Emaad Khansur, Ashish H. Shah ${ }^{2}$, Kyle Lacy, Manish Kuchakulla ${ }^{2}$, Ricardo J. Komotar ${ }^{2}$ \\ 1. 2. Department of Neurological Surgery, University of Miami Miller School of Medicine
}

$\square$ Corresponding author: Ashish H. Shah, ashah@med.miami.edu

Disclosures can be found in Additional Information at the end of the article

\section{Abstract}

Despite surgical resection and adjuvant chemoradiation, survival for glioblastoma remains poor. Because of the dismal prognosis, attention has shifted to alternative adjuvant treatment modalities. Although traditionally limited to systemic malignancies (melanoma, lung and colon cancer), the field of immunotherapy has recently identified glioblastoma as a potential target for new treatments. Anti-tumor vaccines (dendritic cell/heat shock), checkpoint inhibitors, chimeric T-cell receptors, and virotherapy all have been preliminarily trialed in glioblastoma patients with reasonable success and safety. Although there are limitations due to autoimmune reactions and immune escape, immunotherapeutics hold much promise in the future treatment paradigms for malignant glioma.

Categories: Neurosurgery, Oncology

Keywords: immunotherapy, glioblastoma, novel, outcome, clinical trial

\section{Introduction And Background}

Glioblastoma is the most lethal primary central nervous system tumor with an incidence rate of 3.19 per 100,000 person-years, averaging around 13,000 cases diagnosed in the United States per year [1]. Over the last fifteen years, the treatment for glioblastoma multiforme (GBM) included maximal safe surgical resection with combination radiotherapy and adjuvant temozolomide chemotherapy [2]. Despite this treatment, the overall five-year survival still remains poor with an average survival of 14 months after initial diagnosis [2-4]. Although there have been significant advances in understanding the basic pathogenesis of GBM, median survival of patients has changed little in the last 25 years. Because of the dismal prognosis, attention has shifted to alternative adjuvant treatment modalities.

Received 12/29/2017 Review began 01/08/2018 Review ended 01/24/2018 Published 01/30/2018

C) Copyright 2018

Khansur et al. This is an open access article distributed under the terms of the Creative Commons Attribution License CC-BY 3.0., which permits unrestricted use, distribution, and reproduction in any medium, provided the original author and source are credited.
The idea of immunotherapy was first approached by William Coley over 120 years ago when he attempted to increase anti-tumor immune responses by administering bacterial toxins to reduce tumor recurrence. Although his initial attempts were unsuccessful, his research laid the groundwork for potential breakthroughs in the treatment of cancer. Recent research on cancer treatment has been focused on expanding Coley's idea of immunotherapy by utilizing the immune system to target and effectively treat tumors by enhancing either the innate or adaptive immune system. With the Food and Drug Administration's (FDA) approval of Provenge (sipulecel-T, a dendritic cell-based therapy for prostate cancer) and Yerovry (ipilimumab for metastatic melanoma), research interest in immunotherapies in the treatment of cancer has expanded [5]. Current research on glioblastoma focuses on immunotherapy such as vaccines (dendritic cell/heat shock), checkpoint inhibitors, chimeric T-cell receptors, and immunogene 


\section{Cureus}

therapy. See Table 1 for recent clinical trials for malignant glioma over the last five years. We will review the contemporary research on immunotherapeutics for glioblastoma.

\begin{tabular}{|c|c|c|c|c|c|c|}
\hline Name of trial & Type of therapy & Country & Patients & $\begin{array}{l}\text { PFS } \\
\text { (mo) }\end{array}$ & OS (mo) & Year \\
\hline $\begin{array}{l}\text { Phuphanich } \\
\text { et al. [6]. }\end{array}$ & Dendritic Cell & USA & $\begin{array}{l}17 \text { nGBM } 3 \text { rGBM } 1 \\
\text { brainstem glioma }\end{array}$ & $\begin{array}{l}16.9 \\
\text { nGBM }\end{array}$ & 38.4 nGBM & 2013 \\
\hline $\begin{array}{l}\text { Sampson et } \\
\text { al. [7]. }\end{array}$ & Dendritic Cell & USA & 22nGBM & 15.2 & 23.6 & 2011 \\
\hline $\begin{array}{l}\text { Mitchell et } \\
\text { al. [8]. }\end{array}$ & Dendritic Cell & USA & 12nGBM & $>27$ & $>36.6$ & 2015 \\
\hline $\begin{array}{l}\text { Pellegatta et } \\
\text { al. [9]. }\end{array}$ & Dendritic Cell & Italy & 15 rGBM & 4.4 & 8.0 & 2013 \\
\hline $\begin{array}{l}\text { Prins et al. } \\
\text { [10]. }\end{array}$ & Dendritic Cell & USA & 15 nGBM 8 rGBM & - & $\begin{array}{l}35.9 \text { nGBM } \\
17.9 \text { rGBM }\end{array}$ & 2011 \\
\hline $\begin{array}{l}\text { Vik-Mo et al. } \\
\text { [11]. }\end{array}$ & Dendritic Cell & Norway & 7 nGBM & 23.1 & - & 2013 \\
\hline $\begin{array}{l}\text { radul et al. } \\
\text { [12]. }\end{array}$ & Dendritic Cell & USA & 10 nGBM & 9.5 & 28 months & 2011 \\
\hline $\begin{array}{l}\text { Bloch et al. } \\
\text { [13]. }\end{array}$ & Heat Shock & USA & 41 rGBM & 4.8 & 10.7 & 2014 \\
\hline $\begin{array}{l}\text { Crane et al. } \\
\text { [14]. }\end{array}$ & Heat Shock & USA & 12 rGBM & - & 11.8 & 2013 \\
\hline $\begin{array}{l}\text { Brown et al. } \\
\text { [15]. }\end{array}$ & Chimeric antigen T-Cell & USA & 1 rGBM & 7.5 & - & 2016 \\
\hline Ji et al.[16]. & $\begin{array}{l}\text { Adenovirus mutant thymidine } \\
\text { kinase (ADV-TK) }\end{array}$ & China & 53 rGBM & 8.7 & 11.4 & 2015 \\
\hline
\end{tabular}

\section{TABLE 1: Recent immunotherapeutic clinical trial results over the last five years}

nGBM = newly diagnosed glioblastoma multiforme; rGBM = recurrent glioblastoma multiforme; PFS = progression free survival; OS $=$ overall survival

\section{Review}

\section{Vaccine Therapy}

Therapeutic cancer vaccines are designed to eradicate cancer cells by strengthening a patient's own immune response. These vaccines work by activating T-cells (CD4 and CD8) against specific tumor antigens and by inducing an anti-tumoral cellular response by using dendritic 
cells (DC) and heat shock proteins [17].

\section{DC therapy}

DC functions as antigen-presenting cells (APCs) by processing antigens peripherally and presenting them as antigenic peptides to the Tlymphocytes [1]. The development of DC vaccines was predicated on the successful ex vivo culturing of mouse DC's by Inaba, Steinman, and colleagues over 10 years ago. Current preparation of DC vaccines involves exposing the lysate of a patient's tumor to the patient's autologous DCs, which are then treated with a differentiation factor such as GM-CSF. The primed APCs are then injected back into the patient with hopes of generating a T-cell response against the tumor [18]. Recently, DC vaccines have demonstrated some efficacy in improving outcomes for glioblastoma. In a recent systematic review, Bregy et al. demonstrated that autologous DC vaccination improved median OS in patients with newly-diagnosed and recurrent GBM compared to historical trends [19]. Beyond autologous tumor lysate, DC pulsed with specific tumor-associated antigens (TAA) from MAGE1 and AIM-2 demonstrated prolonged survival in newly diagnosed GBM patients [6]. In order to improve the elicited immune response, Mitchell coupled DC vaccination with tetanus/diphtheria(Td) pre-conditioning. The Td toxoid served as a potent recall agent and improved DC migration to lymph nodes. The results of this study showed that there was a markedly enhanced bilateral DC migration that increased both the progression-free survival and overall survival when compared to DC only treated patients [8].

Aside from autologous DC vaccines, allogeneic DC vaccines have also been proposed. A study by Parney and Gustafson (2016) explored the benefits of adding DC therapy with concurrent temozolomide in patients with resected newly diagnosed glioblastoma. DCs were generated from the patient's CD14+ monocytes, pulsed with allogeneic tumor lysate from two patientderived GBM cell cultures, and given to patients during their temozolomide therapy. After vaccination, increased circulating tumor-associated antigen-specific CD8 T-cells were identified, demonstrating that allogenic tumor lysate vaccines are feasible and may generate a tumor antigen-specific immune response [20]. However, there are some inherent concerns of delivering allogeneic lysate to patients including vaccination rejection and lack of antigenic specificity; these concerns are partially mitigated by the fact that allogeneic lysate is derived from multiple cell lines and may be readily available after surgical resection.

In order to explore a new method of delivery, Sayour et al. (2016) examined the efficacy of delivering tumor-derived Ribonucleic acid (RNA) encapsulated in lipophilic nanoparticles in lieu of tumor lysate to systemically activate APCs for induction of therapeutic anti-tumor T-cell immunity. In preclinical murine GBM models, RNA nanoparticles were shown to exceed DCs in mediating anti-tumor activity. These formulations were also shown to be cost-effective and could be formulated expeditiously, providing rapid induction against GBM [21].

\section{Heat shock protein (HSP) vaccines}

The HSP function intracellularly to assemble and transport nascent proteins. HSPs also have a very critical role in the stress response to cellular insult and function by stabilizing proteins and preventing them from aggregating. Therefore, it is thought that they are transcriptionally upregulated in cancer due to increased translation of abnormal protein products. The two major HSP families that have been shown to be released by GBM exosomes are HSP70 and HSP90. The HSP70 family functions to inhibit cell stress-induced apoptotic pathways, facilitate protein folding, and guide protein transport across membranes. The HSP90 family is responsible for protein folding, protein stabilization, and loading onto Major Histocompatibility Complex (MHC) class I molecules. More importantly, HSP90 has been shown to be vital in tumor initiation and proliferation of signaling pathways. Thus, HSPs have been 
shown to have the potential to serve as a way to present tumor-specific antigens to elicit an antitumor immune response [22-25]. Tumor-derived HSPs and other proteins can be complexed together and serve as an antitumor vaccine in patients with glioblastoma. The advantage of these vaccines as compared to others is that HSPs are not targeted to a specific pre-defined antigen but instead to varying types of antigenic proteins upon vaccination, which serves to broadly target the intratumoral heterogeneity that is normally seen in GBM [22, 26-28].

HSP vaccination has generated a robust immune response as well. By binding autologous tumor-derived peptides to HSP-96, Crane et al demonstrated a significant peripheral immune response for the peptides bound to HSP-96 in 11/12 of the patients treated with recurrent GBM. Within this study, immune responders had a median survival of 47 weeks after surgery and vaccination, compared with 16 weeks for the single non-responder. Additionally, inflammatory cytokines (Interferon gamma, CD3 and CD8) were focally increased in the tumor-sites in the immune responder group, suggesting specific immune responses against autologous tumor derived peptides bound to HSP-96 [14]. Other studies seem to confirm the efficacy of HSP-96 vaccination. Bloch et al. (2014) reported a median overall survival of 42.6 weeks after HSP peptide complex-96 vaccination in patients with recurrent glioblastoma. Of note, $66 \%$ of patients in this study were lymphopenic prior to therapy, which is believed to have significantly impacted the anti-tumor immune response. Nevertheless, these studies demonstrate that the HSPPC-96 vaccination may be safe and deserve additional investigation [29].

After encouraging results from the previous phase II trials of HSPPC-96 on glioblastoma, a subsequent multi-institutional trial is being sponsored by Alliance for Clinical Trials of Oncology. This trial is examining whether HSPPC-96 can prolong overall survival in cases of recurrent GBM as an adjuvant therapeutic agent. The study consists of three arms: HSPPC-96 with concomitant bevacizumab, HSPPC-96 with the administration of bevacizumab at tumor progression, and bevacizumab alone. The primary measure of the study is OS with secondary outcomes, evaluating PFS and the safety and tolerability of the combination therapy [22].

\section{Checkpoint Inhibitors}

Immune checkpoints are fundamental in the balance of self-tolerance and immunogenicity. Failed immune checkpoints impede immune responses in refractory cancers that are prone to Tcell anergy and toleragenicity. Programmed cell death protein and ligand (PD-1, PDL-1), metabolic enzymes (e.g., Arginase), and inhibitory immune pathways CTLA4 (Cytotoxic TLymphocyte Associated Antigen 4) have been hypothesized to play a role in immune tolerance. CTLA4, expressed on T-cells, regulates the extent of the T-cell immune response by impeding the CD28 T-cell stimulatory pathway [30]. In the clinical setting, CTLA4 blockade, through use of monoclonal antibodies, increases CD4 T-cell activity, and inhibits regulatory T-cell immunosuppression. In glioma mouse models, systemic blockade of PD-L1 demonstrated longterm survival with concurrent inhibition of regulatory T-cell activity [31]. Furthermore, PD-1 expressed more broadly than CTLA4 in the T-cells in the tumor microenvironment has been found to reduce T-cell activity in the peripheral tissues. Inhibition of PD-1 may augment the effector T-cells, antibody production and NK cell function [32-33]. In the clinical setting, PD-1 blockade demonstrated evidence of anti-tumor immunity in multiple cancers, with less immunotoxicity than with systemic CTLA4 blockade [34]. Additionally, inhibition of its ligand. PD-L1, on tumor cells, may also be a potential target for immunomodulation to prevent interaction with PD-1 receptors (see Figure 1 for major checkpoint inhibition pathways in cancer cells). 


\section{Cureus}

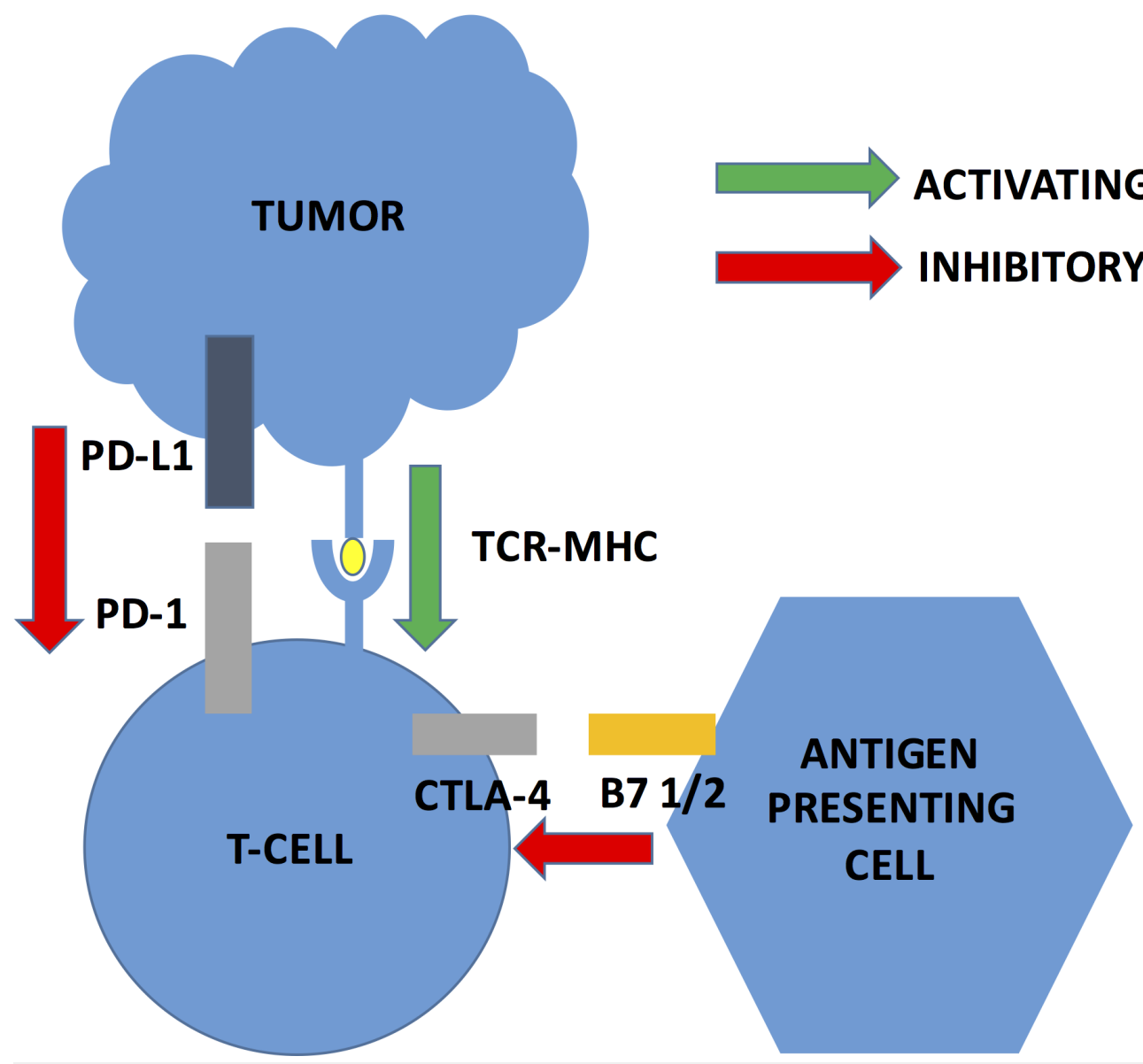

\section{FIGURE 1: Major checkpoint inhibition pathways in cancer cells}

Programmed cell death protein and ligand (PD-1, PDL-1). Cytotoxic T-Lymphocyte Associated Antigen 4 (CTLA4).

Several clinical studies assessing the importance of checkpoint inhibition in glioma have been performed. Berghoff et al. (2014) examined the expression of PDL1 in 135 glioblastoma specimens and noted diffuse or fibrillary PDL1 expression in $88 \%$ of samples from patients with newly diagnosed glioblastoma and in $72 \%$ of samples with recurrent glioblastoma. However, no correlation was found between PDL1 expression and survival [35]. Nevertheless, in animal models, activation of co-stimulatory receptors such as OX40 and blockade of co-inhibitory receptors such as PD1 and CTLA4 induced tumor regression and increased long-term survival [36]. Currently, several clinical trials are ongoing for assessment of monoclonal antibody checkpoint inhibitors (anti-PD-L1 and CTLA-4) for glioblastoma. (NCT02017717, NCT02617589, NCT02529072) Although preliminary results are not yet available, some issues with delivery and brain penetration of these systemic checkpoint inhibitors have been noted.

\section{Chimeric T-cell Receptors (TCR)}

Chimeric antigen receptors (CARs) are a diverse class of receptors that have been created by combining the variable region of an antibody with a T-cell-signaling molecule such as CD3. These newly created receptors are advantageous compared to the TCR-transduced T-cells. CARs have the ability to mimic endogenous TCR-mediated activation without the disadvantages of classical MCH restriction as the antigen recognition site is derived from an antibody. 
Additionally, these antibody directed-CARs can accommodate infinite antigenic diversity and nanomolar antigenic affinity. These receptors can also incorporate costimulator molecules such as CD28 and 4-1BB into the CD3 signaling domain which improves T-cell expansion, survival, and tumor lysis [37-39].

Several studies have demonstrated the safety and preliminary efficacy of this type of therapy in glioblastoma. Brown et al. examined the bioactivity and safety of IL13Ralpha2 redirected chimeric antigen receptor CD8 T-cells in the resection cavity of three patients, and noted transient immune-mediated anti-tumor responses in $2 / 3$ patients with recurrent glioblastoma [40]. Other case reports of similar IL13Ralpha2-directed CAR conducted demonstrated tumor regression and immune responses after intrathecal therapy in patients with multifocal recurrent GBM [41]. Although clear improvements in survival was yet to be shown, this study provided promising results for potential phase 1 human clinical trials of IL13Ralpha2- specific CAR T-cell treatment for GBM.

Additional CAR-mediated treatments have also been investigated. O’ Rourke et al. (2016) utilized autologous T-cells re-directed to the EGFR variant III mutation in nine patients with recurrent glioblastoma. This study positively demonstrated a significant expansion of CARTEGFRvIII cells one week after infusion and tumor infiltration by activated CAR T-cells. This study showed that EGFRvIII-CAR therapy was safe without the evidence of off-target toxicity or cytokine release [41]. These studies helped establish a foundation that adoptive CAR T-cell therapy can be applied to the treatment of glioblastoma.

\section{Viroimmunotherapy}

The use of viruses to mediate gene immunotherapy in the treatment of tumors is a promising approach and has a wide variety of applications. Treatments can include transferring genes for inflammatory proteins to tumor cells, inhibition of immunosuppressing tumor genes, or transferring proinflammatory and tumor antigen genes to professional antigen presenting cells. Previous clinical trials have focused on conditional cytotoxicity and oncolytic viruses, which may induce a secondary immune response by generating foreign antigens and producing a proinflammatory immune beacon in tumor cells [42-43]. Several clinical trials using adenovirus, herpes simplex virus, replicating retroviruses have been conducted with preliminary results demonstrating survival benefit [16]. Many of these viral-based therapies utilize intraparenchymal convection-enhanced delivery methods to deliver the vector into the surgical cavity, and are now undergoing early phase I/II clinical trials [16, 44-46].

\section{Pitfalls}

While current research on the various treatments of glioblastoma has provided some encouraging results, each therapy has associated drawbacks. Clinical translation of DC vaccines has been modest due to limitations imposed by the source of antigens, poor DC maturation, tumor-mediated immunosuppression, and allergic encephalomyelitis [20]. The limitations of heat shock vaccines include the acquisition of adequate tissue for vaccine production and limited inclusion criteria (requiring near complete tumor resection) [22]. Both vaccination approaches are limited by the quality of the tumor lysate and the antigenic presentation; if tumor lysate is not representative of the heterogenic tumor or if the lysate does not induce antigen response, vaccination may not confer a benefit.

Other molecular immunotherapeutics are also limited in certain regards. The use of checkpoint inhibitors has potential adverse effects including severe autoimmune reactions which may include colitis, pneumonitis, hypophysitis, and hepatotoxicity [47]. Immune checkpoint inhibitors cannot function unless its target receptors (PD-1, CTLA-4) exist in the tumor microenvironment. Additionally, robust inhibition of the checkpoint pathway may result in 
uncontrolled systemic autoimmune reactions. In order to avoid this potential limitation, checkpoint inhibitors may need to be delivered intracranially to bypass systemic immune reactions. For chimeric antigen receptors, T-cell generation may limit treatment, and once delivered, antigens may be lost as the GBM adapts and modifies its antigen presentation by the glioblastoma [15].

\section{Conclusions}

The burgeoning field of immunotherapy holds much promise for the treatment of glioblastoma. Novel biologics and pharmaceuticals are evolving treatment paradigms. The recent research in vaccine therapy, check-point inhibitors, chimeric antigen T-cell receptors, and viroimmuno therapy has provided an opportunity to supplement the current treatment of glioblastoma potentially, improving prognosis and overall survival for these patients. Although there are several barriers to an effective safe treatment, future larger prospective studies may help elucidate the role of immunotherapy in these patients.

\section{Additional Information}

\section{Disclosures}

Conflicts of interest: In compliance with the ICMJE uniform disclosure form, all authors declare the following: Payment/services info: All authors have declared that no financial support was received from any organization for the submitted work. Financial relationships: All authors have declared that they have no financial relationships at present or within the previous three years with any organizations that might have an interest in the submitted work. Other relationships: All authors have declared that there are no other relationships or activities that could appear to have influenced the submitted work.

\section{References}

1. Reardon DA, Mitchell DA: The development of dendritic cell vaccine-based immunotherapies for glioblastoma. Semin Immunopathol. 2017, 39:225-239. 10.1007/s00281-016-0616-7

2. Stupp R, Hegt ME, Mason WP, et al.: Effects of radiotherapy with concomitant and adjuvant temozolomide versus radiotherapy alone on survival in glioblastoma in a randomised phase III study: 5-year analysis of the EORTC-NCIC trial. Lancet Oncol. 2009, 10:459-66. 10.1016/S1470-2045(09)70025-7

3. Coffey RJ, Lunsford LD, Taylor FH: Survival after stereotactic biopsy of malignant gliomas . Neurosurgery. 1988, 22:465-7. 10.1227/00006123-198803000-00003

4. Ma X, Lv Y, Liu J, et al.: Survival analysis of 205 patients with glioblastoma multiforme: clinical characteristics, treatment and prognosis in China. J Clin Neurosci. 2009, 16:1595-8. 10.1016/j.jocn.2009.02.036

5. Kamran N, Calinescu A, Candolfi M, et al.: Recent advances and future of immunotherapy for glioblastoma. Expert Opin Biol Ther. 2016, 16:1245-64. 10.1080/14712598.2016.1212012

6. Phuphanich S, Wheeler CJ, Rudnick JD, et al.: Phase I trial of a multi-epitope-pulsed dendritic cell vaccine for patients with newly diagnosed glioblastoma. Cancer Immunol Immunother. 2013, 62:125-35. 10.1007/s00262-012-1319-0

7. Sampson JH, Aldape KD, Archer GE, et al.: Greater chemotherapy-induced lymphopenia enhances tumor-specific immune responses that eliminate EGFRvIII-expressing tumor cells in patients with glioblastoma. Neuro Oncol. 2011, 13:324-33. 10.1093/neuonc/noq157

8. Mitchell DA, Batich KA, Gunn MD, et al.: Tetanus toxoid and CCL3 improve dendritic cell vaccines in mice and glioblastoma patients. Nature. 2015, 519:366-9. 10.1038/nature14320

9. Pellegatta S, Eoli M, Frigerio S, et al.: The natural killer cell response and tumor debulking are associated with prolonged survival in recurrent glioblastoma patients receiving dendritic cells loaded with autologous tumor lysates. Oncoimmunology. 2013, 2:23401. 10.4161/onci.23401

10. Prins RM, Soto H, Konkankit V, et al.: Gene expression profile correlates with T-cell infiltration and relative survival in glioblastoma patients vaccinated with dendritic cell 
immunotherapy. Clin Cancer Res. 2011, 17:1603-15. 10.1158/1078-0432.CCR-10-2563

11. Vik-Mo EO, Nyakas M, Mikkelsen BV, et al.: Therapeutic vaccination against autologous cancer stem cells with mRNA-transfected dendritic cells in patients with glioblastoma. Cancer Immunol Immunother. 2013, 62:1499-509. 10.1007/s00262-013-1453-3

12. Fadul CE, Fisher JL, Hampton TH, et al.: Immune response in patients with newly diagnosed glioblastoma multiforme treated with intranodal autologous tumor lysate-dendritic cell vaccination after radiation chemotherapy. J Immunother. 2011, 34:382-9.

10.1097/CJI.0b013e318215e300

13. Bloch O, Crane CA, Fuks Y, et al.: Heat-shock protein peptide complex-96 vaccination for recurrent glioblastoma: a phase II, single-arm trial. Neuro Oncol. 2014, 16:274-9. 10.1093/neuonc/not203

14. Crane CA, Han SJ, Ahn B, et al.: Individual patient-specific immunity against high-grade glioma after vaccination with autologous tumor derived peptides bound to the $96 \mathrm{KD}$ chaperone protein. Clin Cancer Res. 2013, 19:205-14. 10.1158/1078-0432.CCR-11-3358

15. Brown CE, Alizadeh D, Starr R, et al.: Regression of glioblastoma after chimeric antigen receptor T-cell therapy. N Engl J Med. 2016, 375:2561-9. 10.1056/NEJMoa1610497

16. Ji N, Weng D, Liu C, et al.: Adenovirus-mediated delivery of herpes simplex virus thymidine kinase administration improves outcome of recurrent high-grade glioma. Oncotarget. 2016, 7:4369-78. 10.18632/oncotarget.6737

17. Guo C, Manjili MH, Subjeck JR, Sarkar D, Fisher PB, Wang XY: Therapeutic cancer vaccines: past, present, and future. Adv Cancer Res. 2013, 119:421-75. 10.1016/B978-0-12-4071902.00007-1

18. Shah P, Goldberg J: Novel approaches to pediatric cancer: immunotherapy . AIMS Medical Science. 2015, 2:104-11. 10.3934/medsci.2015.2.104

19. Bregy A, Wong TM, Shah AH, Goldberg JM, Komotar RJ: Active immunotherapy using dendritic cells in the treatment of glioblastoma multiforme. Cancer Treat Rev. 2013, 39:891-907. 10.1016/j.ctrv.2013.05.007

20. Parney IF, Gustafson MP, Peterson T, Steinmetz SM, Dietz AB: Allogeneic tumor lysate/autologous dendritic cell vaccines in newly diagnosed glioblastoma:Clinical Trial MC1272. Neuro Oncol. 2016, 18:24-25. 10.1093/neuonc/nox168.109

21. Sayour E, De Leon G, Pham C, Grippin A, Flores C, Mitchell D: Translatable RNA nanoparticles supplant dendritic cell vaccines in cellular immunotherapy. Neuro Oncol. 2016, 18:89. 10.1093/neuonc/now212.372

22. Ampie L, Choy W, Lamano JB, Fakurnejad S, Bloch O, Parsa AT: Heat shock protein vaccines against glioblastoma: from bench to bedside. J Neurooncol. 2015, 123:441-8. 10.1007/s11060015-1837-7

23. Tosti G, Cocorocchio E, Pennacchioli E, Ferrucci PF, Testori A, Martinoli C: Heat-shock proteins-based immunotherapy for advanced melanoma in the era of target therapies and immunomodulating agents. Expert Opin Biol Ther. 2014, 14:955-67.

10.1517/14712598.2014.902928

24. Oki Y, Younes A: Heat shock protein-based cancer vaccines. Expert Rev Vaccines. 2004, 3:403-11. 10.1586/14760584.3.4.403

25. Takakura Y, Takemoto S, Nishikawa M: Hsp-based tumor vaccines: state-of-the-art and future directions. Curr Opin Mol Ther. 2007, 9:385-91.

26. Winograd EK, Ciesielski MJ, Fenstermaker RA: Novel vaccines for glioblastoma: clinical update and perspective. Immunotherapy. 2016, 8:1293-1308. 10.2217/imt-2016-0059

27. van Ommeren R, Staudt MD, Xu H, Hebb MO: Advances in HSP27 and HSP90-targeting strategies for glioblastoma. J Neurooncol. 2016, 127:209-19. 10.1007/s11060-016-2070-8

28. Sayegh ET, Oh T, Fakurnejad S, Bloch O, Parsa AT: Vaccine therapies for patients with glioblastoma. J Neurooncol. 2014, 119:531-46. 10.1007/s11060-014-1502-6

29. Svedlund J, Sjodin I: A psychosomatic approach to treatment in the irritable bowel syndrome and peptic ulcer disease with aspects of the design of clinical trials. Scand J Gastroenterol Suppl. 1985, 109:147-51. 10.3109/00365528509103950

30. Grosso JF, Jure-Kunkel MN: CTLA-4 blockade in tumor models: an overview of preclinical and translational research. Cancer Immun. 2013, 13:5.

31. Wainwright DA, Chang AL, Dey M, et al.: Durable therapeutic efficacy utilizing combinatorial blockade against IDO, CTLA-4, and PD-L1 in mice with brain tumors. Clin Cancer Res. 2014, 20:5290-301. 10.1158/1078-0432.CCR-14-0514 
32. Dong H, Zhu G, Tamada K, Chen L: B7-H1, a third member of the B7 family, co-stimulates Tcell proliferation and interleukin-10 secretion. Nat Med. 1999, 5:1365-9. 10.1038/70932

33. Toomer KH, Chen Z: Autoimmunity as a double agent in tumor killing and cancer promotion . Front Immunol. 2014, 5:116. 10.3389/fimmu.2014.00116

34. Pardoll DM: The blockade of immune checkpoints in cancer immunotherapy . Nat Rev Cancer. 2012, 12:252-64. 10.1038/nrc3239

35. Berghoff AS, Kiesel B, Widhalm G, et al.: Programmed death ligand 1 expression and tumorinfiltrating lymphocytes in glioblastoma. Neuro Oncol. 2015, 17:1064-75.

10.1093/neuonc/nou307

36. Zeng J, See AP, Phallen J, et al.: Anti-PD-1 blockade and stereotactic radiation produce longterm survival in mice with intracranial gliomas. Int J Radiat Oncol Biol Phys. 2013, 86:343-9. 10.1016/j.ijrobp.2012.12.025

37. Sampson JH, Choi BD, Sanchez-Perez L, et al.: EGFRvIII mCAR-modified T-cell therapy cures mice with established intracerebral glioma and generates host immunity against tumorantigen loss. Clin Cancer Res. 2014, 20:972-84. 10.1158/1078-0432.CCR-13-0709

38. Sengupta S, Mao G, Gokaslan ZS, Sampath P: Chimeric antigen receptors for treatment of glioblastoma: a practical review of challenges and ways to overcome them. Cancer Gene Ther. 2016, 24:121-9. 10.1038/cgt.2016.46

39. Samaha H, Naggar SEl, Ahmed N: Armed hunter killers: discerning the role of adoptive T-cell transfer for glioblastoma. Immunotherapy. 2015, 7:481-5. 10.2217/imt.15.22

40. Brown CE, Badie B, Barish ME, et al.: Bioactivity and safety of IL13Ralpha2-redirected chimeric antigen receptor CD8+ T cells in patients with recurrent glioblastoma. Clin Cancer Res. 2015, 21:4062-72. 10.1158/1078-0432.CCR-15-0428

41. O'Rourke DM, Nasrallah M, Morrissette JJ, et al.: Pilot study of T cells redirected to EGFRvIII with a chimeric antigen receptor in patients with EGFRvIII+ glioblastoma. J Clin Oncol. 2016, 17:110-111.

42. Parney IF, Chang LJ: Cancer immunogene therapy: a review . J Biomed Sci. 2003, 10:37-43. 10.1159/000068081

43. Glick RP, Lichtor T, Cohen EP: Cytokine immunogene therapy . Neurosurg Focus. 2000, 9:e2.

44. Markert JM, Medlock MD, Rabkin SD, et al.: Conditionally replicating herpes simplex virus mutant, G207 for the treatment of malignant glioma: results of a phase I trial. Gene Ther. 2000, 7:867-74. 10.1038/sj.gt.3301205

45. Lang FF, Bruner JM, Fuller GN, et al.: Phase I trial of adenovirus-mediated p53 gene therapy for recurrent glioma: biological and clinical results. J Clin Oncol. 2003, 21:2508-18. 10.1200/JCO.2003.21.13.2508

46. Geletneky K, Huesing J, Rommelaere J, et al.: Phase I/IIa study of intratumoral/intracerebral or intravenous/intracerebral administration of Parvovirus H-1 (ParvOryx) in patients with progressive primary or recurrent glioblastoma multiforme: ParvOryx01 protocol. BMC Cancer. 2012, 12:99. 10.1186/1471-2407-12-99

47. Preusser M, Lim M, Hafler DA, Reardon DA, Sampson JH: Prospects of immune checkpoint modulators in the treatment of glioblastoma. Nat Rev Neurol. 2015, 11:504-14.

10.1038/nrneurol.2015.139 